\title{
NETWORK CODING PERFORMANCE FOR RELIABLE MULTICAST
}

\author{
Majid Ghaderi, Don Towsley and Jim Kurose \\ Department of Computer Science \\ University of Massachusetts Amherst \\ \{mghaderi, towsley, kurose\} ecs. umass. edu
}

\begin{abstract}
The capacity gain of network coding has been extensively studied in wired and wireless networks. Recently, it has been shown that network coding improves network reliability by reducing the number of packet retransmissions in lossy networks. However, the extent of the reliability benefit of network coding is not known. This paper quantifies the reliability gain of network coding for reliable multicasting in a wireless network where network coding is the most promising. We define the expected number of transmissions per packet as the performance metric for reliability and derive analytical expressions characterizing the performance of network coding. For a tree-based multicast, we derive expressions for the expected number of transmissions at the source of the multicast and inside the multicast tree. We also analyze the performance of error control mechanisms based on rateless codes and automatic repeat request (ARQ). We then use the analytical expressions to study the impact of multicast group size on the performance of different error control schemes. Our numerical results show that network coding significantly reduces the number of retransmissions in lossy networks compared to end-to-end ARQ scheme, however, rateless coding and link-by-link $A R Q$ are able to achieve performance results comparable to that of network coding. Interestingly, link-by-link $A R Q$ can outperform rateless coding depending on the network size and loss probability. We conjecture that network coding achieves a logarithmic reliability gain with respect to multicast group size compared to a simple ARQ scheme.
\end{abstract}

\section{INTRODUCTION}

In traditional networks, data packets are carried by store-and-forward mechanisms in which the intermediate nodes (relays or routers) forward an exact copy of data packets that they have received. With network coding, a network node is allowed to combine several packets that it has generated or received into one or several outgoing packets. The original paper of Ahlswede et al. [1] showed the utility of network coding for multicast in wireline networks. Recently, network coding has been applied to wireless networks and received significant attention as a means of improving network capacity and coping with unreliable wireless links [2], [3]. In fact, the unreliability and broadcast nature of wireless links make wireless networks a natural setting for network coding.

In spite of significant research on the capacity gain of network coding, the reliability gain of network coding is largely unknown. In this paper, we study the application of network coding as an error control technique for reliable multicasting in a wireless network. Our goal is to quantify the benefit of using network coding compared to traditional error control techniques such as ARQ and rateless coding.

We consider tree-based reliable multicast with four different error control techniques. A brief description of these error control techniques is as follows:

1) End-to-end ARQ: The root of the multicast tree retransmits each packet until it is correctly received by all the multicast receivers. All other nodes in the tree only forward packets they receive from their parents to their children.

2) End-to-end FEC: This technique is commonly referred to as rateless coding [4]. Similar to endto-end ARQ, only the root of the multicast tree is responsible for retransmitting a packet until it is received by all receivers. All other nodes only forward the packets they receive from their parents to their children. For FEC-based schemes, we assume the use of a block coding technique to create coded packets for transmission.

3) Link-by-link ARQ: Every node of the multicast tree is responsible for reliable transmission of packets to its children. That is, a node retransmits the packet it has received from its parent to its children until the packet is correctly received by all of its children. Note that some children may receive more than a copy of the packet because of the random nature of packet losses.

4) Link-by-link FEC: Throughout this paper, we refer to this technique as network coding. With 
network coding, every node is responsible for reliable delivery of packets it has received from its parent to its children. In a sense, with network coding, each node performs rateless coding to deliver a packet to its children.

The rest of the paper is organized as follows. In Sections II and III, we analyze end-to-end and linkby-link error control techniques, respectively, and derive exact expressions for the expected number of transmissions at the source and within the multicast tree. In Section IV, we provide numerical examples to illustrate the performance of different error control techniques by evaluating the expressions we derive in Sections II and III. Our conclusions as well as future work are discussed in Section V.

\section{END-TO-END ERROR CONTROL}

Throughout this paper, we make the following assumptions, although it is straightforward to extend our results to more general cases:

- Each node of the multicast tree has exactly $K$ children,

- All transmissions are wireless broadcasts,

- Packet losses over all links are independent and occur with probability $p$ (Bernouli loss process),

- There is reliable and instantaneous feedback.

\section{A. Probability Distribution of the Number of Transmis-} sions

Let $N_{r}$ denote the number of transmissions of a packet to the root of a subtree of height $r$ (from its parent) before the packet is received by all nodes of the subtree. For the source of a multicast tree of height $h$, we interpret $N_{h}$ as the number of packet transmissions at the source until the packet is received by all the multicast receivers (see Figure 1).

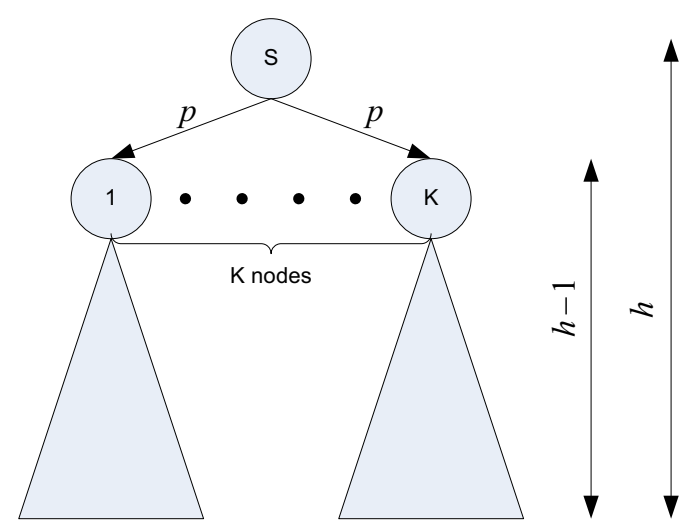

Fig. 1. Tree topology for reliable multicast.
Define $F_{r}(i)$ as follows:

$$
F_{r}(i)=\mathbb{P}\left\{N_{r} \leq i\right\}, \quad 0 \leq r \leq h, i \geq 0
$$

where, $F_{r}(0)=0$ according to the definition.

Similar to [5], we develop recursive equations to compute $F_{r}(i)$ in the case of ARQ and FEC. First, consider the case $r>0$ and denote the root of the subtree by $s$. The probability that $j$ packets out of $i$ packets that have been transmitted to node $s$ are received by node $s$ is given by a binomial distribution,

$$
\mathbb{P}\{j \mid i\}=\left(\begin{array}{l}
i \\
j
\end{array}\right)(1-p)^{j} p^{i-j}, \quad 0 \leq j \leq i .
$$

Note that for the root of the multicast tree the error probability is zero, i.e., $p=0$, and hence $\mathbb{P}\{j \mid i\}=1$, if $j=i$, and $\mathbb{P}\{j \mid i\}=0$, otherwise. If node $s$ receives $j$ packets, it will broadcast the $j$ received packets to its children. For each child, the probability that all nodes of the subtree rooted at that child receive a packet is given by $F_{r-1}(j)$. Since the children of a node have independent packet losses, the probability that all the nodes of the subtrees rooted at children of node $s$ receive a packet is given by $\left\{F_{r-1}(j)\right\}^{K}$, which we denote by $F_{r-1}^{K}(j)$ for notational simplicity. Therefore, by summing over all possible values of $j$, we obtain

$$
F_{r}(i)=\sum_{j=0}^{i}\left(\begin{array}{l}
i \\
j
\end{array}\right)(1-p)^{j} p^{i-j} F_{r-1}^{K}(j), \quad 0<r<h .
$$

Hence, we have a recursive equation for computing $F_{r}(i)$ for $r>0$. Interestingly, computing $F_{r}(j)$ for $r>0$ is independent of the end-to-end error control technique.

Next, we compute $F_{0}(i)$ for the leaves of the multicast tree as follows:

1) End-to-End ARQ:

The probability that a (leaf) node does not receive any packet out of $i$ transmitted packets is given by $p^{i}$. Therefore, with probability $1-p^{i}$ the node receives at least one copy of the packet. Therefore,

$$
F_{0}(i)=1-p^{i} .
$$

2) End-to-End FEC:

We assume the block size for coding is $B$. Clearly, $F_{0}(i)=0$ for $i<B$. Hence, we consider $i \geq B$ in the following. The probability that a node receives at least $B$ coded packets out of $i$ transmitted packets is given by a binomial distribution. Therefore,

$$
F_{0}(i)=\sum_{j=B}^{i}\left(\begin{array}{l}
i \\
j
\end{array}\right)(1-p)^{j} p^{i-j}, \quad i \geq B .
$$


So far, we have determined $F_{r}(i)$ for all subtrees of height $r$. As mentioned before, for the root of the multicast tree, we have $\mathbb{P}\{i \mid i\}=1$. Hence, the expression $F_{h}(i)$ can be simplified as follows

$$
F_{h}(i)=F_{h-1}^{K}(i),
$$

where, $F_{h-1}(i)$ is given by (3).

\section{B. Expected Number of Transmissions}

For the root of the multicast tree, the expected number of transmissions until a packet is received by all receivers is as follows.

1) End-to-End ARQ:

$$
\mathbb{E}\left[N_{h}\right]=\sum_{i=0}^{\infty}\left(1-F_{h}(i)\right)=1+\sum_{i=1}^{\infty}\left(1-F_{h}(i)\right) .
$$

2) End-to-End FEC:

$$
\mathbb{E}\left[N_{h}\right]=\frac{1}{B} \sum_{i=0}^{\infty}\left(1-F_{h}(i)\right)=1+\frac{1}{B} \sum_{i=B}^{\infty}\left(1-F_{h}(i)\right) .
$$

Next, we compute the expected number of transmissions in the multicast tree (not just at the root) until a packet is received by all receivers.

Let $T_{h}$ denote the total number of transmissions in the multicast tree until a packet is received by all receivers. First, we compute the expected number of transmissions in the tree per transmission at the root of the multicast tree. Let $X_{r}$ denote the number of transmissions in a subtree of height $r$ per each transmission at the root of the subtree. Then,

$$
\begin{aligned}
X_{r} & =1+\sum_{j=0}^{K}\left(\begin{array}{c}
K \\
j
\end{array}\right)(1-p)^{j} p^{K-j}\left(j X_{r-1}\right) \\
& =1+K(1-p) X_{r-1},
\end{aligned}
$$

where,

$$
X_{0}=0 .
$$

It is therefore obtained that

$$
X_{r}=\frac{(K q)^{r}-1}{K q-1},
$$

where $q=1-p$. Therefore, the expected number of transmissions per packet in the multicast tree is given by

$$
\begin{aligned}
\mathbb{E}\left[T_{h}\right] & =X_{h} \mathbb{E}\left[N_{h}\right] \\
& =\frac{(K q)^{h}-1}{K q-1} \mathbb{E}\left[N_{h}\right] .
\end{aligned}
$$

Note that if $K q=1$ then it is simply obtained that

$$
\mathbb{E}\left[T_{h}\right]=h \mathbb{E}\left[N_{h}\right] .
$$

\section{LINK-BY-LINK ERROR CONTROL}

For link-by-link error control, we consider a simple topology as depicted in Figure 2 in which a source $s$ broadcasts a packet to all of its $K$ children. Let $N$ denote the number of transmissions of a packet by the source until the packet is received by all $K$ children. Define $F(i)$ as follows:

$$
F(i)=\mathbb{P}\{N \leq i\},
$$

where $F(0)=0$.

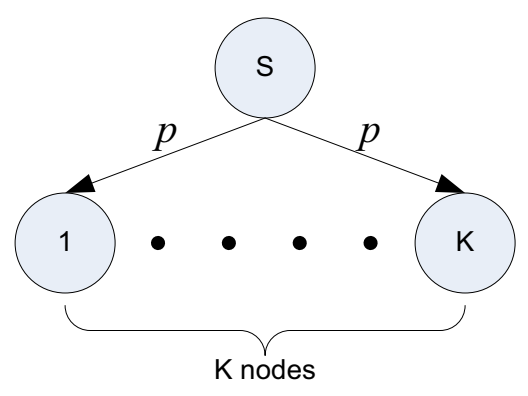

Fig. 2. Model for link-by-link error control.

\section{A. Probability Distribution of the Number of Transmis- sions}

Interestingly, $F_{0}(i)$ from the previous section can be used to compute the expected number of transmissions in the case of link-by-link error control. In particular, we obtain the following expressions.

1) Link-by-Link ARQ:

The probability that a child does not receive any packet out of $i$ transmitted packets is given by $p^{i}$. Therefore, with probability $1-p^{i}$ the child receives at least a copy of the packet. All $K$ children of a node are independent, therefore, the probability that every child receives at least a packet is given by

$$
F(i)=\left(1-p^{i}\right)^{K}
$$

2) Network Coding:

We assume the block size for network coding is $B$ and $i \geq B$. Clearly, $F(i)=0$ for $i<B$. The probability that a child receives at least $B$ coded packets out of $i$ transmitted packets is given by a binomial distribution. Therefore, the probability 
that every child is able to receive enough packets to recover the original $B$ packets is given by

$$
F(i)=\left\{\sum_{j=B}^{i}\left(\begin{array}{l}
i \\
j
\end{array}\right)(1-p)^{j} p^{i-j}\right\}^{K} .
$$

\section{B. Expected Number of Transmissions}

Similar to the previous section, the expected number of transmissions at the root of the multicast tree is simply given by

1) Link-by-Link ARQ:

$$
\mathbb{E}[N]=\sum_{i=0}^{\infty}(1-F(i))=1+\sum_{i=1}^{\infty}(1-F(i)) .
$$

2) Network Coding:

$$
\mathbb{E}[N]=\frac{1}{B} \sum_{i=0}^{\infty}(1-F(i))=1+\frac{1}{B} \sum_{i=B}^{\infty}(1-F(i)) .
$$

Next, consider a multicast tree of height $h$. At height $r$ of the tree, there are $K^{h-r}$ nodes. For each of them, the expected number of transmissions is given by $\mathbb{E}[N]$ because of the link-by-link error control mechanism. Let $T_{h}$ denote the total number of transmissions in the tree. It is obtained that

$$
\mathbb{E}\left[T_{h}\right]=\mathbb{E}[N] \sum_{r=1}^{h} K^{h-r}=\frac{K^{h}-1}{K-1} \mathbb{E}[N] .
$$

Note that for $K=1$, we have

$$
\mathbb{E}\left[T_{h}\right]=h \mathbb{E}[N] .
$$

\section{NUMERICAL EXAMPLES}

We have numerically evaluated the expected number of transmissions at the source and in the multicast tree for different tree heights, block sizes and error probabilities. A summary of our numerical results is presented in this section. For FEC-based error control techniques, i.e., end-to-end FEC and network coding, we have assumed the use of block codes with block length $B$.

As a base for comparison, in Figure 3, we have plotted the expected number of transmissions for $h=1$. This is the case of having a source transmitting packets to $K$ receivers in its transmission range. The block size $B$ is set to 16 . We have also generated results for larger values of $B$ which exhibit the same behavior in the number of transmissions, and hence are not presented here. Since feedback overhead is ignored, ARQ is indeed the optimal error control technique in a non-coded case.

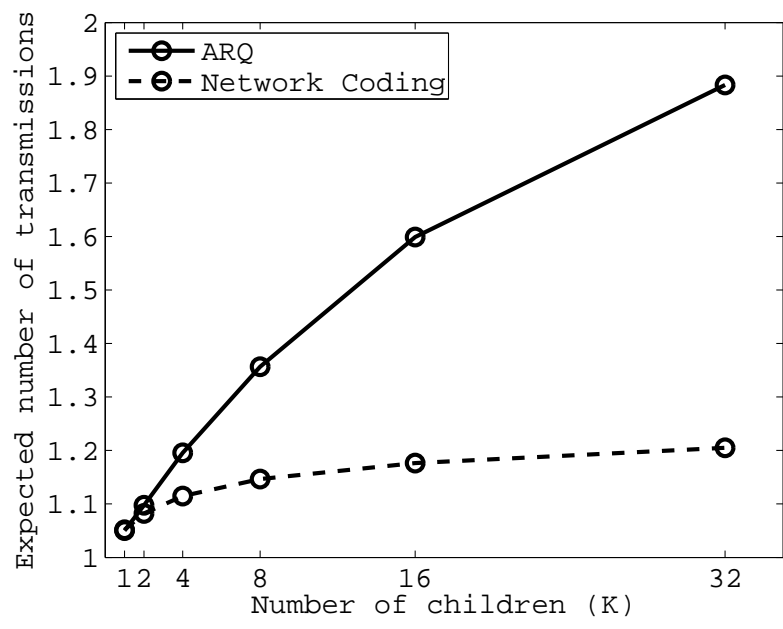

(a) $p=0.05$

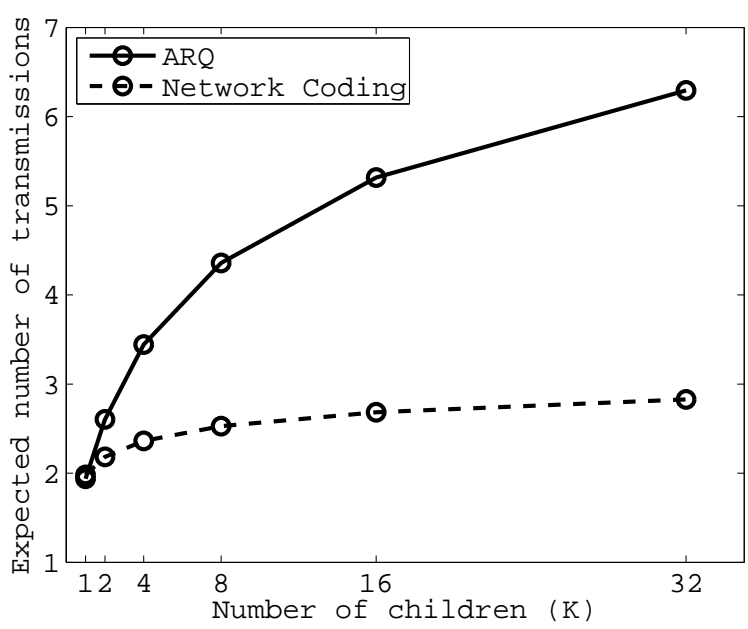

(b) $p=0.5$

Fig. 3. The single op case: number of source transmission versus number of children for $p=0.05$ and $p=0.5$.

Nevertheless, network coding outperforms ARQ in both low-loss and high-loss regimes as shown in Figures 3(a) and 3(b), respectively.

In the following subsections, we will study the impact of different parameters such as $h, B$ and $K$ on the performance of different error control mechanisms.

\section{A. Impact of Tree Height (h)}

Next, we look at multicast trees with $h=2,4$. In both cases network coding outperforms all the other techniques. Interestingly, link-by-link ARQ and end-toend FEC show different behavior with different tree heights. Figures 4 and 5 depict the expected number of transmissions for a tree of height $h=2$ and error probabilities $p \in\{0.05,0.5\}$. It can be seen from the 


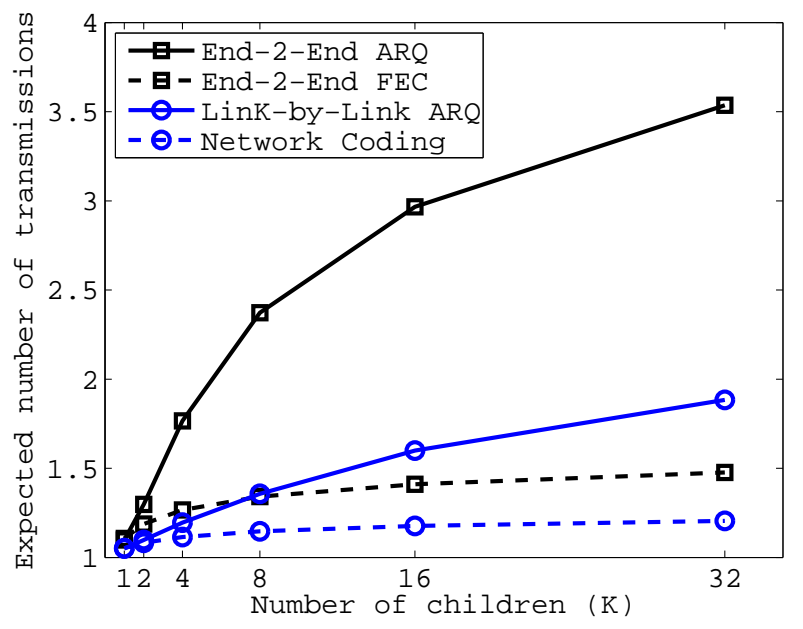

(a) No. of transmissions at source

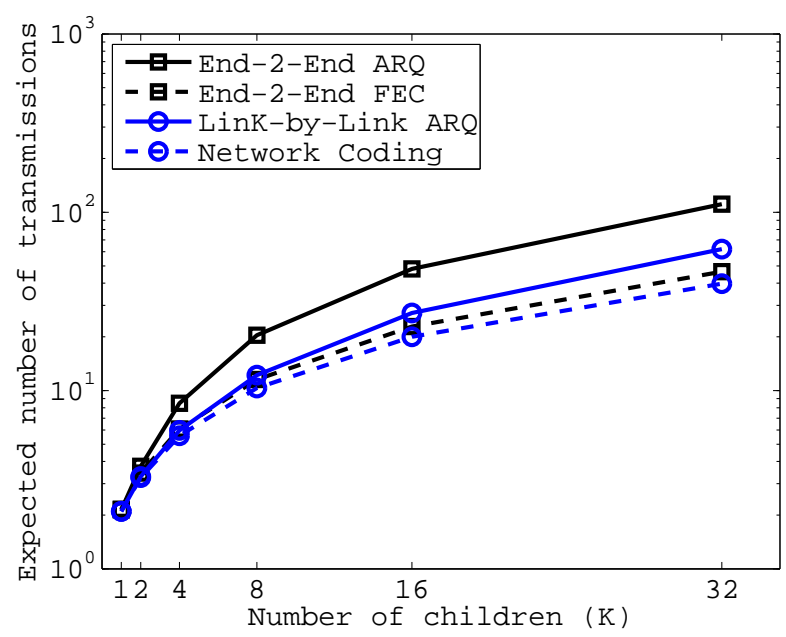

(b) No. of transmissions in tree

Fig. 4. Expected number of transmissions (at source, and in tree) versus number of children, $p=0.05$ and $h=2$.

figures that, in some cases, end-to-end FEC has better performance than link-by-link ARQ although the difference is not significant. Interestingly, there is a significant difference between end-to-end ARQ (dominant error control technique in Internet) and network coding. In particular, when $p=0.5$ representing a high-loss regime, the difference in the number of transmissions at the source is extremely large.

Figures 6 and 7 show the number of transmissions for a tree of height $h=4$. Interestingly, link-by-link error control techniques always outperform end-to-end techniques. Moreover, the difference between end-to-end ARQ and network coding is significant even for small values of $K$. Again, in high-loss regimes, there is a huge difference between end-to-end ARQ and network coding.

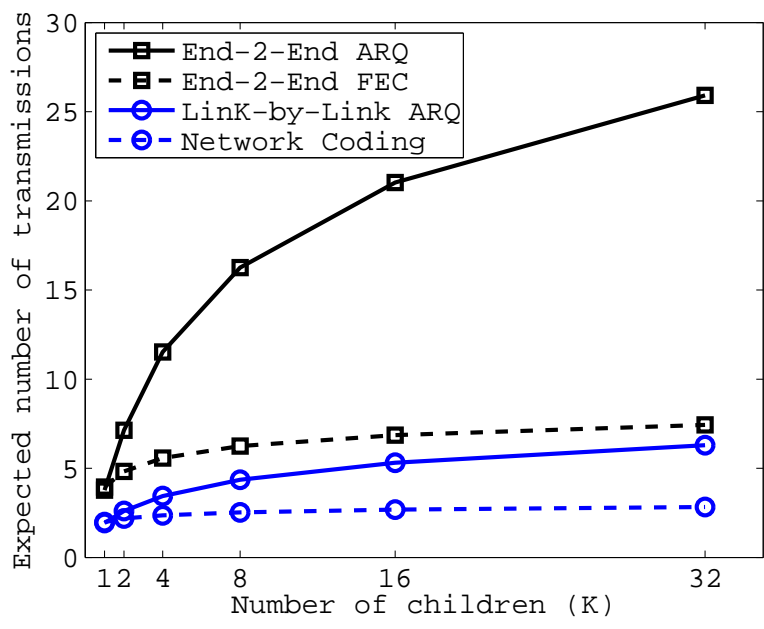

(a) No. of transmissions at source

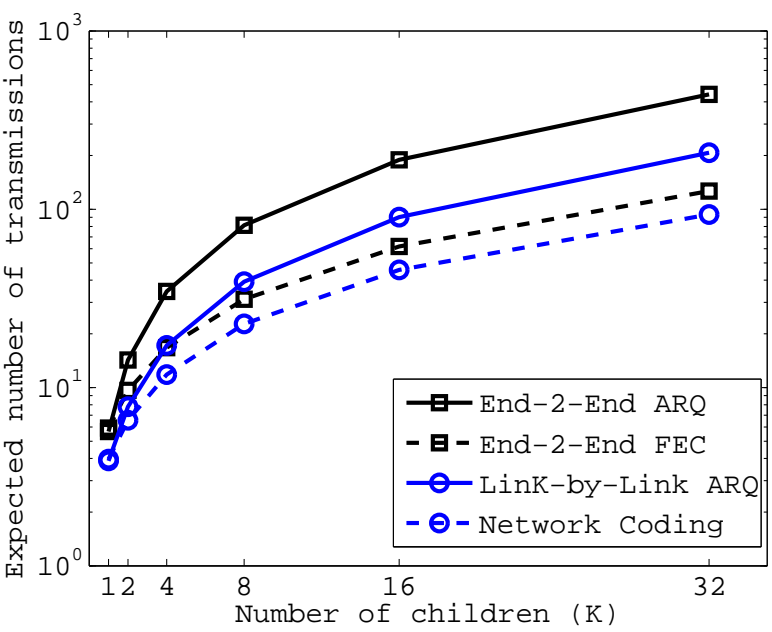

(b) No. of transmissions in tree

Fig. 5. Expected number of transmissions (at source, and in tree) versus number of children, $p=0.5$ and $h=2$.

\section{B. Impact of Block Size (B)}

Intuitively, as $B$ increases, the expected number of transmissions decreases. The best performance is achieved when $B \rightarrow \infty$. In this case, based on the law of large numbers, the expected number of transmissions approaches a constant number that depends on the loss probability. Figure 8 shows the expected number of transmissions for $B=32,512,1024$. The loss probability is set to $p=0.05,0.5$ and the network topology is depicted in Figure 2. It can be seen from the figure that by increasing $B, \mathbb{E}[N]$ approaches a constant value. The limiting value of $\mathbb{E}[N]$ is equal to the expected number of transmissions at $K=1$. In particular, the limit is $\frac{1}{1-p}$ which evaluates to 1.05 and 2 for $p=0.05$ and $p=0.5$, 


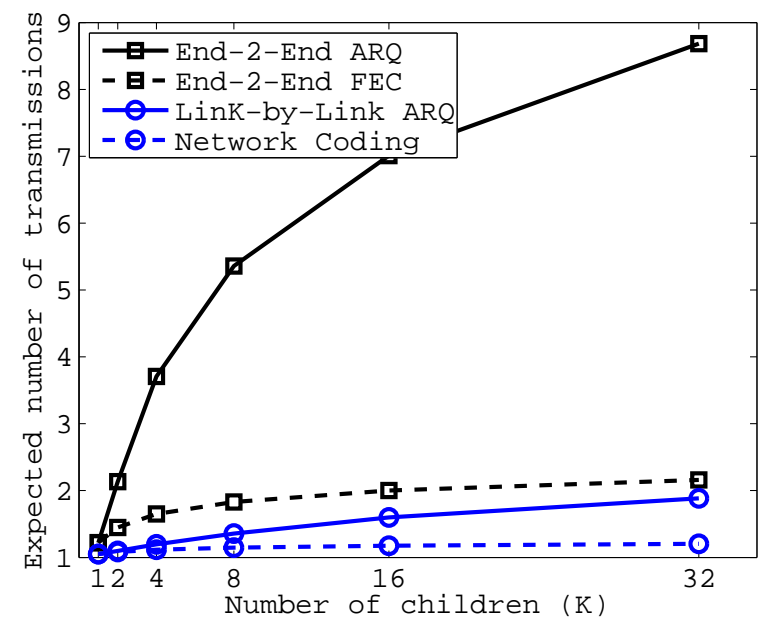

(a) No. of transmissions at source

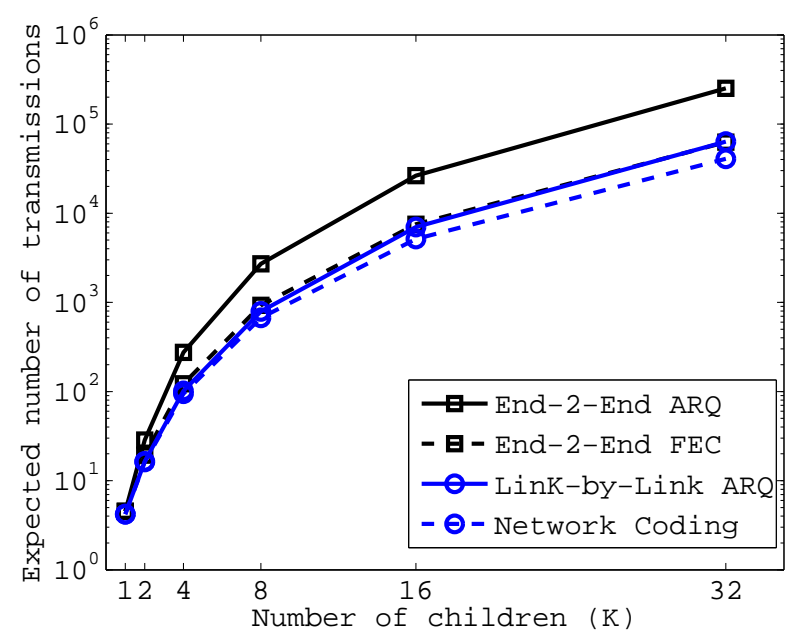

(b) No. of transmissions in tree

Fig. 6. Expected number of transmissions (at source, and in tree) versus number of children, $p=0.05$ and $h=4$.

respectively. Interestingly, $\mathbb{E}[N]$ converges to limit very fast. This indicates that in practice a moderate size block is sufficient to obtain the full reliability benefit available via network coding.

\section{Impact of Group Size (K)}

Now consider the network topology of Figure 2 with ARQ, i.e., $B=1$. Clearly, as $K$ increases, the expected number of transmissions increases as well. However, the rate of increase is not linear as we saw in previous figures. In Figure 9 we have plotted $\mathbb{E}[N]$ for a wide range of $K$ and different loss probabilities. Notice that the horizontal axis shows $\log _{2}(K)$ and hence, $K$ ranges from 1 to $2^{20}$ in the figure. There are three curves corresponding to loss probabilities of $p=0.05,0.3,0.5$.

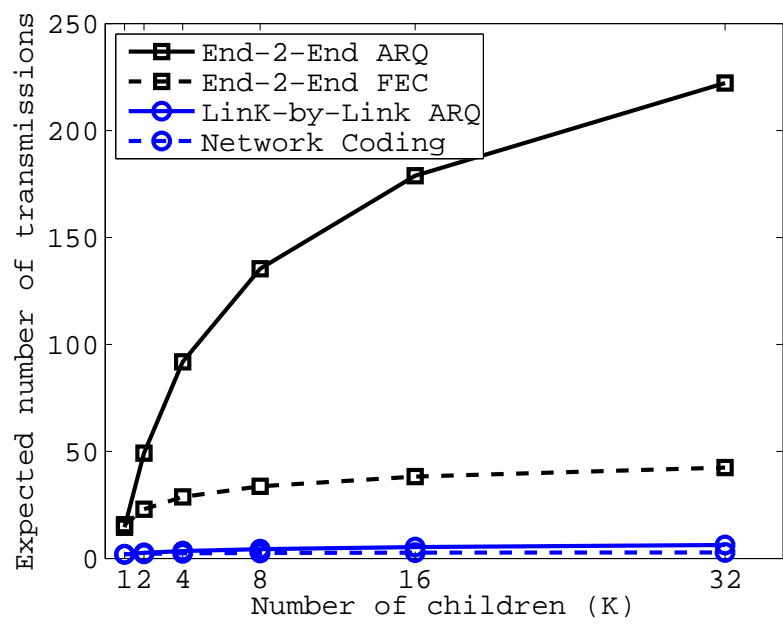

(a) No. of transmissions at source

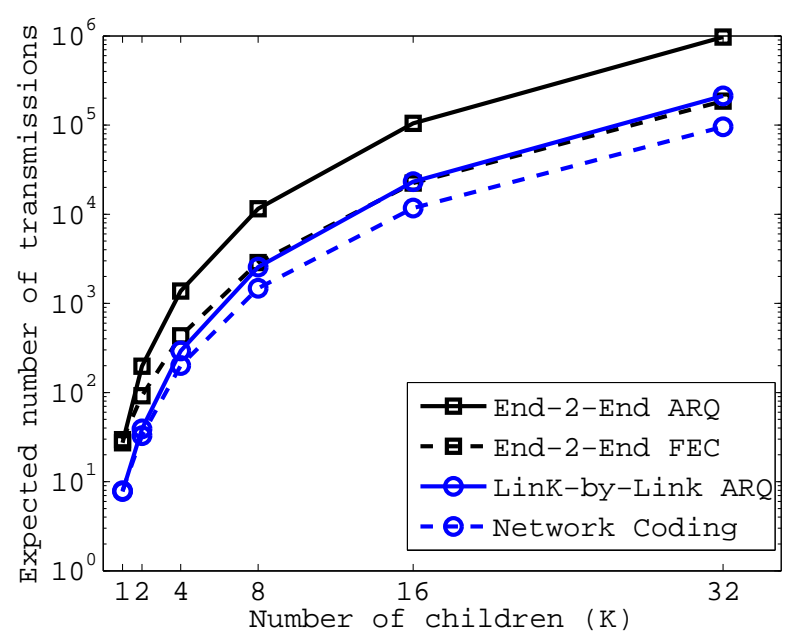

(b) No. of transmissions in tree

Fig. 7. Expected number of transmissions (at source, and in tree) versus number of children, $p=0.5$ and $h=4$.

Interestingly, the three curves are very close to straight lines especially for large values of $K$, e.g., $K>2^{5}$. This indicates that as $K \rightarrow \infty, \mathbb{E}[N] \rightarrow \log (K)$.

Based on the results and discussion presented in previous subsections, we conjecture that the benefit of network coding, defined as the reduction in the expected number of transmissions compared to ARQ, would be $\log (K)$ in the limit as $B$ and $K$ grow to infinity.

\section{CONClusion}

In this paper, we studied the reliability benefits of network coding for tree-based reliable multicasting. Four types of error control techniques, namely, end-to-end ARQ, end-to-end FEC, link-by-link ARQ and network coding were considered. We analyzed the expected 


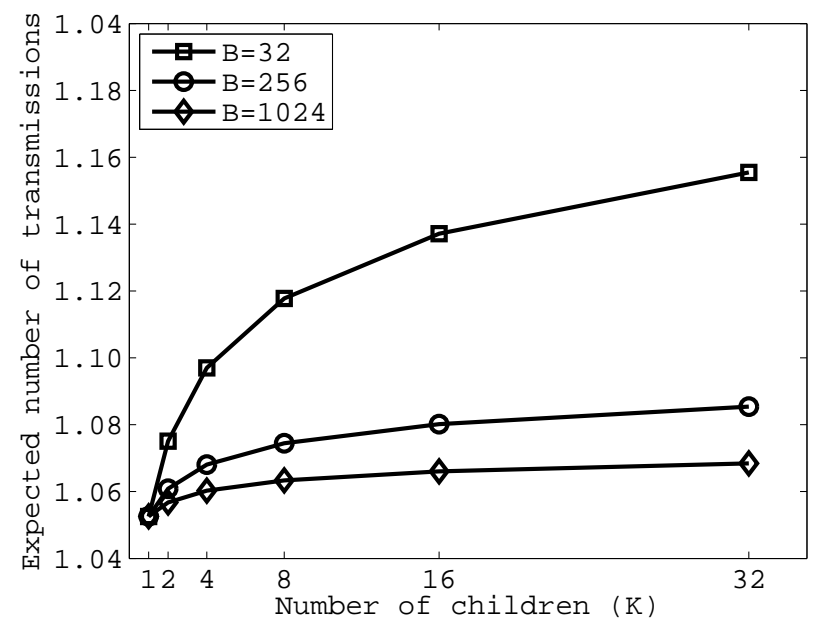

(a) $p=0.05$

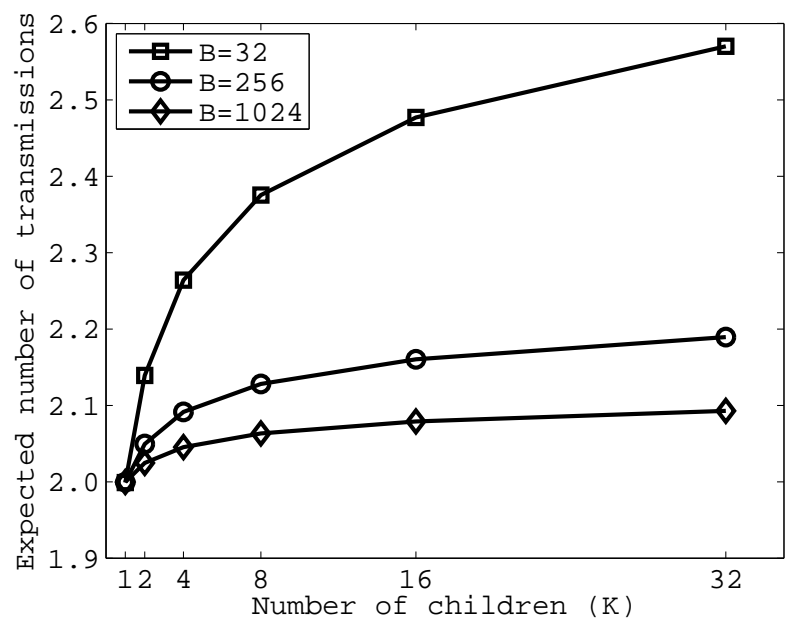

(b) $p=0.5$

Fig. 8. Impact of block size $B$.

number of transmissions at the source and within the multicast tree as the performance metrics of interest. We derived expressions for the expected number of transmissions and utilized them to study the impact of different parameters such as block size, loss probability and multicast group size on the performance of different error control techniques. We found that network coding significantly reduces the number of retransmissions in lossy networks compared to end-to-end ARQ scheme; however, rateless coding and link-by-link ARQ are able to achieve performance results comparable to that of network coding. Based on our numerical results, we hypothesize that network coding achieves a logarithmic reliability gain with respect to multicast group size compared to a simple ARQ scheme. In the future, we

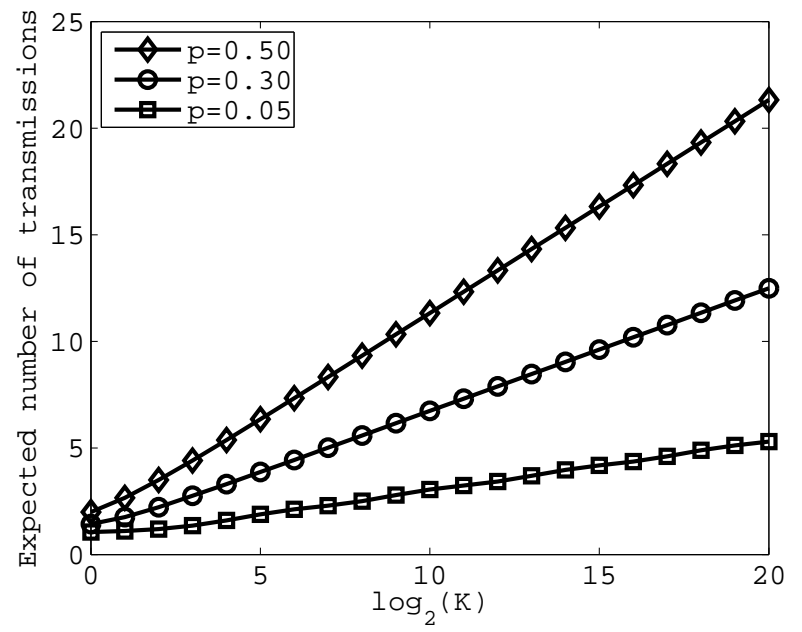

Fig. 9. Impact of group size $K$.

would like to extend our analysis to more complicated multicast topologies such as a grid with a significant amount of path diversity.

\section{ACKNOWLEDGEMENTS}

This research was supported by DARPA CBMANET and US/UK ITA programs. This research is continuing through participation in the International Technology Alliance sponsored by the U.S. Army Research Laboratory and the U.K. Ministry of Defence and was accomplished under Agreement Number W911NF-06-3-0001. The views and conclusions contained in this document are those of the authors and should not be interpreted as representing the official policies, either expressed or implied, of the US Army Research Laboratory, the U.S. Government, the UK Ministry of Defense, or the UK Government. The US and UK Governments are authorized to reproduce and distribute reprints for Government purposes notwithstanding any copyright notation hereon.

\section{REFERENCES}

[1] R. Ahlswede, N. Cai, S.-Y. R. Li, and R. W. Yeung, "Network information flow," IEEE Transactions on Information Theory, vol. 46, no. 4, pp. 1204-1216, July 2000.

[2] D. S. Lun, M. Medard, and M. Effros, "On coding for reliable communication over packet networks," in Proc. Allerton, Urbana Champaign, USA, September 2004.

[3] S. Katti, H. Rahul, W. Hu, D. Katabi, M. M. Médard, and J. Crowcroft, "XORs in the air: Practical wireless network coding," in Proc. ACM SIGCOMM, Pisa, Italy, September 2006.

[4] J. Byers, M. Luby, M. Mitzenmacher, and A. Rege, "A digital fountain approach to reliable distribution of bulk data," in Proc. ACM SIGCOMM, Vancouver, Canada, February 1998.

[5] P. Bhagwat, P. P. Mishra, and S. K. Tripathi, "Effect of topology on performance of reliable multicast communications," in Proc. IEEE INFOCOM, Toronto, Canada, June 1994, pp. 602-609. 